\title{
A method for the construction of initial structures for molecular dynamics simulations of nanocrystals with nonequilibrium grain boundaries containing extrinsic dislocations
}

\author{
A. A. Nazarov ${ }^{\dagger}$, R. T. Murzaev \\ †aanazarov@imsp.ru \\ Institute for Metals Superplasticity Problems of RAS, 39 Khalturin street, Ufa, 450001, Russia
}

\begin{abstract}
A method for the construction of initial atomic models of nanocrystals with extrinsic grain boundary dislocations (EGBDs) in grain boundaries (GBs) for molecular dynamics (MD) simulations is developed. The method is realized for f.c.c. nanocrystals with columnar grains having common crystallographic axis [112] parallel to the column axis and thus divided only by [112] tilt GBs. This system is convenient for studies of interactions between GBs and lattice dislocations, since each grain can be deformed by edge dislocations of only one slip system, which have lines parallel to the [112] axis. In order to introduce extrinsic dislocations to the boundaries of a selected grain, its contour is assumed to be strained by a given shear strain $\gamma$ so that a contour of a freely sheared grain is formed. This contour is filled in by atoms of a f.c.c. lattice with [112] direction parallel to the column axis and then the grain thus formed is subjected to an elastic shear strain $-\gamma$. This results in a deformed grain having the original shape, on the boundaries of which precursors of EGBDs are formed. In order to prevent these precursors from spontaneous annihilation during MD relaxation, one can temporarily fix GB atoms, or apply a proper external stress, or do both. A case study is carried out using two different protocols of MD relaxation to determine atomic structures and energies of nonequilibrium GBs.
\end{abstract}

Keywords: nonequilibrium grain boundary, extrinsic grain boundary dislocations, energy, molecular dynamics.

УДК: 548.4:620.186.8

\section{Метод построения исходных структур для молекулярно- динамического моделирования нанокристаллов с неравновесными границами зерен, содержащими внесенные дислокации}

\author{
Назаров А. А. ${ }^{\dagger}$, Мурзаев Р. Т. \\ Институт проблем сверхпластичности металлов РАН, ул. Халтурина, 39, Уфа, 450001, Россия
}

\begin{abstract}
Разработан метод построения исходных атомных моделей нанокристаллов с внесенными зернограничными дислокациями (ВЗГД) в границах зерен (ГЗ) для молекулярно-динамического моделирования. Метод реализован для случая г.ц.к. нанокристаллов с колончатыми зернами, имеющими общее кристаллографическое направление [112], параллельное оси колонны, и таким образом разделенными только границами наклона [112]. Эта система удобна для исследования взаимодействий между ГЗ и решеточными дислокациями, так как каждое зерно в ней может деформироваться скольжением краевых дислокаций только одной системы скольжения, линии которых параллельны направлению [112]. Для того чтобы создать внесенные дислокации в границах выбранного зерна, мысленно допускается, что его контур деформирован сдвигом на заданную величину $\gamma$, так что образуется контур свободно деформированного зерна. Этот контур заполняется атомами г.ц.к. решетки с направлением [112], параллельным оси колонны. Затем образованное таким образом зерно деформируется упругим сдвигом на $-\gamma$. В результате образуется деформированное зерно, имеющее исходную форму, в границах которого формируются прекурсоры ВЗГД. Чтобы препятствовать самопроизвольной аннигиляции этих прекурсоров при молекулярно-динамической релаксации, можно временно фиксировать атомы в ГЗ, приложить соответствующее внешнее напряжение или одновременно сделать и то, и другое. Проведен анализ конкретного примера с использованием двух разных протоколов молекулярно-динамической релаксации, определены атомные структуры и энергии неравновесных ГЗ.
\end{abstract}

Ключевые слова: неравновесная граница зерен, внесенная зернограничная дислокация, энергия, молекулярная динамика. 


\section{Introduction}

During low-temperature plastic deformation of polycrystalline materials their grain boundaries (GBs) act as strong barriers for slip and accumulate extrinsic grain boundary dislocations (EGBDs) [1,2]. This results in a specific structure of GBs, which is commonly referred to as the nonequilibrium structure [3-5]. Nonequilibrium GBs induce long-range internal stress fields and possess an enhanced specific energy that results in their different properties and effect on the properties of polycrystals as compared to equilibrium GBs typical for well annealed materials $[2,4]$. For instance, nonequilibrium GBs have an enhanced diffusion coefficient [6,7], GB sliding ability [2], significantly influence the mechanical [8] and even electronic properties of polycrystals [9].

The nonequilibrium GB structure is typical for the structure of bulk nanostructured materials processed by severe plastic deformation (SPD) methods [10,11]. Electron microscopic observations showed that after SPD the GBs had a specific strain contrast indicating on high internal stresses in grains [12], while differential scanning calorimetric studies revealed an excess energy release due to the GB recovery without a grain growth [13].

Starting from the most general concepts concerning the interaction of GBs with lattice dislocations during plastic flow, Rybin and co-authors [14] developed a theory of fragmentation of materials, i.e., grain subdivision, which is a basis of SPD-processing of metallic materials. Basing on this theory, dislocation and disclination models of nonequilibrium GB structure in nanostructured materials were proposed [15-17]. These models allowed one to evaluate some important structural and energetic parameters of SPD-processed nanomaterials such as root mean square elastic strain and excess GB energy.

A more direct study of the properties of nonequilibrium GBs and their effect on the properties of polycrystals requires the knowledge of their atomic structure, for which molecular dynamics (MD) simulations are needed. A key part of this study is a construction of appropriate atomistic models, which include necessary configurations of defects to study.

In the literature, several atomistic studies of nonequilibrium GB structures in bicrystals and nanocrystals have been reported recently [18-20]. In these studies, however, the nonequilibrium structure was either introduced into GBs by pseudo-random local shifts of GB atoms from their equilibrium positions [18] and creating free volumes [19], or was assumed to occur simply due to the Voronoi construction $[18,20]$. However, these kinds of initial structures do not capture the main features of nonequilibrium GBs such as the existence of EGBDs, which are the only sources of long-range stress fields of GBs. So far, by authors' knowledge, there have been no atomistic studies of nonequilibrium GBs containing EGBDs.

In the present work, a method for the construction of atomic models of nanocrystals with nonequilibrium GBs containing EGBDs is proposed on an example of a nickel columnar nanocrystal with the column axis [112]. A case study of the structures of nanocrystals using two different protocols of $\mathrm{MD}$ relaxation is carried out and the excess energy of GBs for these structures is calculated.

\section{Plastic strain of a grain and formation of EGBDs}

In order to elucidate the idea behind the construction method, imagine an infinitely elongated grain with a square shaped cross section, which can be plastically deformed by slip on the planes of a single slip system parallel to the horizontal plane (Fig. 1a). The grain is not isolated but surrounded by other grains as a whole considered as an effective matrix grain. The only role of this effective grain is to make the probe grain a part of bulk material and form boundaries with the grain under consideration, which are non-transparent for lattice dislocations.

Assume now that the grain is deformed plastically under an applied simple shear $\gamma$, while the matrix deforms only elastically. Then dislocations causing the strain of the island grain will cross the latter and be trapped by its boundaries

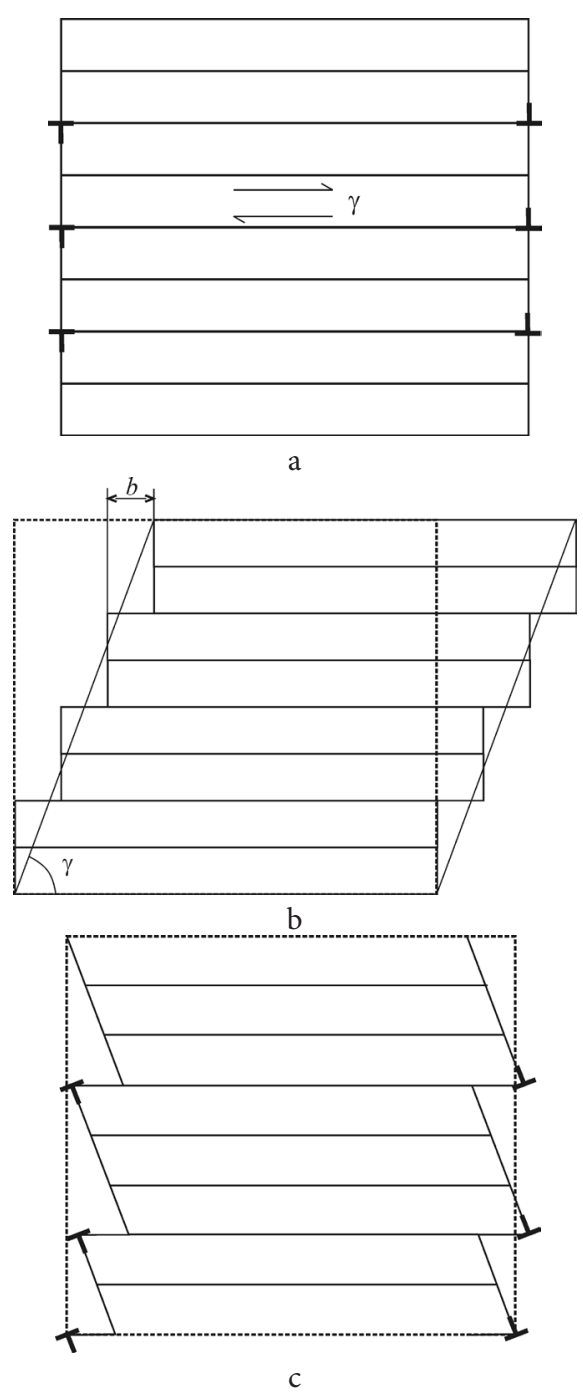

Fig. 1. Schematics of the formation of dislocations in the boundaries of a probe grain surrounded by a matrix due to a simple shear of the grain (a), distortion of an isolated grain by the same shear (b) and formation of EGBD "precursors" by an opposite elastic shear (c). 
thus forming EGBDs as shown in Fig. 1a. Definitely, this will result in the formation of a metastable nonequilibrium structure of the GBs, which is the cause of long range elastic strain fields around the boundaries in both the probe and matrix grains and an excess energy of elastic nature.

Now imagine that the grain is taken out from its place and deformed freely by the same simple shear $\gamma$. This will result in a plastic distortion of the grain as shown in Fig. 1b, i.e. the grain will have no elastic strains but steps on its surfaces are formed due to the emergence of dislocations. If we take this plastically distorted grain as an initial system, subject it to an elastic shear of opposite sign $-\gamma$ and put back inside its previous place, the surface steps will be located in the GBs and become "precursors" of EGBDs (Fig. 1c).

The main difference of these EGBD precursors from real EGBDs formed due to the constrained slip as in Fig. 1a is that during their creation all elastic strain has been localized in the probe grain, while in the system of Fig. 1a it is distributed in the strain field of dislocations, i.e. in both the probe and matrix grains. Therefore, special atomic relaxation procedures should be applied to the structure obtained by the creation of EGBD precursors to redistribute the elastic strain fields.

\section{Construction of initial structure for [112] columnar f.c.c. nanocrystals}

The idea of introducing dislocations in GBs for a construction of atomic models is the most conveniently realized for the case of quasi-two-dimensional systems, i.e. for nanocrystals consisting of columnar grains infinitely elongated in one direction. A particularly convenient and physically meaningful system of this kind is the one consisting of f.c.c. grains with the column axis [112]. When the applied stress has only components normal to this axis, the grains will deform by slip of only edge dislocations with the Burgers vector $\frac{1}{2}[\overline{1} 10]$ and lines parallel to [112]. This greatly facilitates the detailed atomistic analysis of the processes of dislocation-GB interactions. Recently, such a system has been used by Shimokawa et al. [21] to study the interaction of [112] tilt GBs with lattice dislocations.

Let us consider a computational cell containing a columnar f.c.c. nanocrystal schematically presented in Fig. 2. The cell consists of four grains, which have a common crystallographic axis [112], the direction of which coincides with that of the $z$-axis. The grains have a regular hexagon shape cross section with an edge $a$. Maximum size of the hexagons, $d=2 a$, will be taken as the grain size.

The grains of the system can be given arbitrary orientations with a crystallographic direction [112] parallel to the $z$-axis. As an example, consider a specific case, when grains 1 and 2 have orientations of slip planes parallel to $x \mathrm{Oz}$ and $y \mathrm{Oz}$ planes, respectively, and grains 3 and 4 are rotated to angles $\pm 20^{\circ}$ from the orientation of grain 1 to form a symmetric high-angle tilt GB between each other.

In order to construct a nanocrystal with equilibrium GBs, the grains are directly filled in by atoms belonging to crystal lattices of corresponding orientations. The system thus obtained is checked to find the pairs of too closely spaced atoms in $\mathrm{GB}$ regions. In these pairs, one of the atoms is

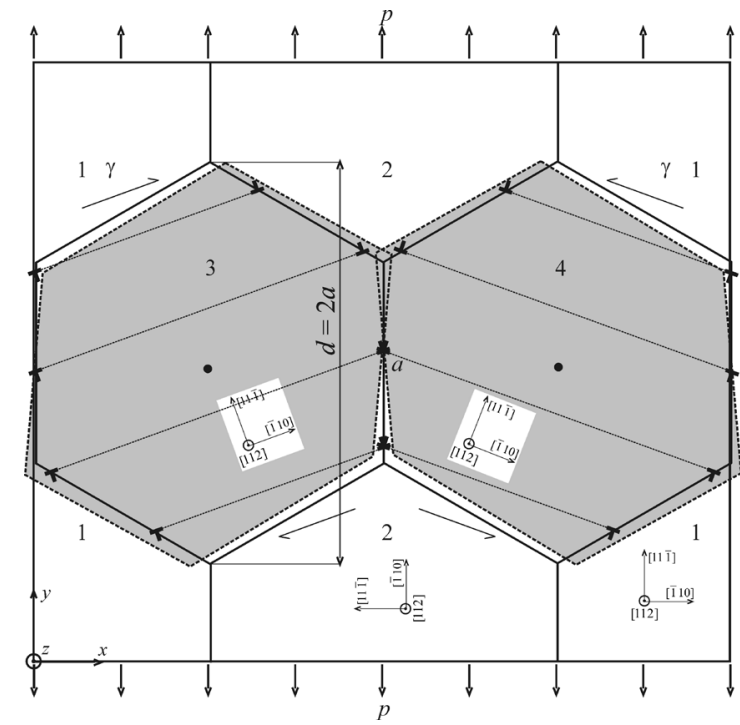

Fig. 2. Schematic rendering of the computation cell for columnar f.c.c. nanocrystals with a [112] axis and the formation of EGBDs due to shear strains of its two grains.

eliminated to avoid too strong repulsive interactions during the start of simulations.

Construction of initial structures with nonequilibrium GBs is carried out according to the idea described in the previous section. Grains 3 and 4, provided that they are isolated, will be distorted into the regions highlighted by a grey color in Fig. 2, if some simple shear strain $\gamma$ by slip of dislocations in (111) slip planes is applied to each of them. In order to obtain corresponding grains with EGBDs, one has simply to distort crystallites with perfect lattices occupying the gray regions into the ones provided for grains 3 and 4, respectively. In fact, there is no need in an explicit definition of the distorted grain regions. Instead, one can fill in larger regions by atoms belonging to lattices of appropriate orientations, then apply shear distortions $-\gamma$ with respect to the centers of grains 3 and 4 and then select only those atoms, which belong to these grains. Finally, we will obtain grains 3 and 4 containing precursors of EGBDs schematically depicted in Fig. 2.

For symmetric mutual orientations of grains 3 and 4 the EGBDs will be located symmetrically with respect to the planes of GBs between these grains. Despite the uniform strains of grains, distributions of EGBDs along host GBs can be not exactly uniform, since crystallography can impose some irregularities. Such irregularities are typical for grain boundary dislocations in periodic grain boundaries [22]. In the present research, we will not be interested in exact positions of individual EGBDs in the GBs but will consider the EGBD systems formed as mesodefects, which are responsible for long-range internal stress fields and enhanced elastic energy. Also, we will not calculate the exact values of EGBD densities, which are different on different GBs, but will characterize the degree of nonequilibrium by the value of shear distortion $\gamma$. Knowing this parameter, one can easily calculate the density of EGBDs for any of the GBs of the deformed grains. Varying the values of $\gamma$, nanocrystals with different degrees of GB nonequilibrium can be created. 


\section{Simulation procedures}

Pairs of opposite signed dislocations formed by the above described construction method are attracted to each other and will immediately annihilate, if one starts energy minimization without precautions. In order to avoid this, several atomic relaxation protocols can be used.

1. One can select GB atoms and temporarily fix them at their positions unless the other part of the system relaxes to an equilibrium, then remove the restrictions and carry out a full relaxation.

2. An appropriate external stress to the computation cell can be applied in order to induce shear stresses that will clamp the dislocations to GBs.

3. One can combine the fixing of GB atoms with the application of external stress.

Each of these procedures allows one to redistribute the elastic strain field of EGBD precursors to make it as more as similar to the one of real EGBD systems. This will fix the dislocations to their metastable positions and further relaxation without constraints will lead to fully relaxed structures of nanocrystals with the GB defects.

For case studies, protocols 1 and 2 have been used to simulate a nanocrystal with the GB nonequilibrium created by shear strain $\gamma=0.10$.

Molecular dynamics simulations were carried out for nickel using embedded atom method (EAM) potential developed in [23]. Corresponding lattice parameter is equal to $a_{0}=0.352 \mathrm{~nm}$ and cohesion energy to $\varepsilon=4.45 \mathrm{eV}$. The grain size of nanocrystals was set equal to $d=15 \mathrm{~nm}$. The period of computation cell along [112] axis was equal to $h_{z}=3 a_{0} / 2|[112]|=1.293 \mathrm{~nm}$. Periodic boundary conditions with constant pressure were applied along all directions (zero along $x$ and $z$ and zero or $p$ along $y$ ).

Energy minimization was done by molecular dynamics code XMD [24]. Time step of 2 fs was used overall.

When using Protocol 1, atoms having energies higher that $-4.20 \mathrm{eV}$ were chosen for fixing. This allowed one to select 3642 high energy atoms among 69426 atoms in total. Visualization has shown that all these atoms are located in GBs and, depending on a particular GB, belong to layers with thickness of 1 to 2 interatomic distances. Note that the choice of the energy level conventionally dividing low and high energy atoms can be one more parameter by varying which one can try to obtain different final relaxed structures from the same initial ones. Applying this constraint, 4000 steps of relaxation were done followed by 20000 steps without constraints.

In Protocol 2, tensile stress $p=7 \mathrm{GPa}$ was applied along the $y$-axis and the system relaxed for 2000 steps under this stress. Then the stress value decreased to zero by decrements of 1 per cent after each 100 steps. Finally, 10000 steps of relaxation were applied at zero external stress.

In both protocols, intermediate atomic structures were recorded to an XMOL file in order to visualize the structure changes during relaxation.

Atomic structures were visualized by visualization codes RasMol [25] and OVITO [26].

\section{Simulation results and discussion}

Video files demonstrating the processes of relaxation by protocols 1 and 2 are presented in supplementary files Relax_1.mp4 and Relax_2.mp4, respectively. In these videos, common neighbor analysis [27] has been used to visualize atoms with different neighborhoods, so the dislocation and GB cores (white atoms) and stacking faults (red atoms) can be seen. The videos show that during relaxation the GBs emit a few partial dislocations into grains that results in a partial relaxation of the GB nonequilibrium structure. These dislocations in some cases glide across the whole grain and are trapped by opposite GBs, in other cases the dislocations stop in the grains. Different numbers of dislocations at different positions are generated in the two ways of relaxation used and this allows one to assume that somehow different relaxed structures are obtained.

Presented in Fig. 3a-c are the atomic energy maps for the equilibrium structure of nanocrystal, when no EGBDs are introduced into GBs, and for nonequilibrium structures obtained by the two simulation protocols.

In the equilibrium structure, all atoms in grain interiors have energies close to the energy of atoms in a perfect lattice, $-4.45 \mathrm{eV}$. All high-energy atoms are located at GBs, therefore, elastic strains in this nanocrystal are localized in a thin layer with the thickness of about a couple interatomic distances, i.e. have a short-range character. This is typical for equilibrium GBs. On the contrary, energy maps for the nanocrystals with nonequilibrium GBs show the presence of high energy atoms in the grains even far from GBs. Therefore, elastic strains induced by these GBs cover the distances much larger than the crystallographic width of GBs, i.e. have a long-range character. This is a characteristic feature of nonequilibrium GBs $[4,11]$. Visually, the energy levels are higher in the case of using the second protocol of relaxation, i.e. when applying a temporary external stress (Fig. 3b). This is confirmed by a calculation of the average energy of defects per unit area of GBs. The total area of GBs per simulation cell is equal to $S=6 d h_{z}$ and, dividing the energy of defects to this value, one can obtain $\gamma_{g b}=1.279 \mathrm{Jm}^{-2}$ for the equilibrium nanocrystal and $1.469 \mathrm{Jm}^{-2}$ and $1.563 \mathrm{Jm}^{-2}$ for the nonequilibrium nanocrystals relaxed by Protocols 1 and 2, respectively. Since only a couple of partial dislocations are located in grains, all these defect energies can be attributed to GBs. Correspondingly, excess energies of nonequilibrium GBs, i.e. the differences between their energies and that of the equilibrium GB, are equal to $\gamma_{e x}=0.190$ and $0.284 \mathrm{Jm}^{-2}$ for the two nonequilibrium nanocrystals, respectively.

An inspection of Fig. $3 \mathrm{~b}$ and $\mathrm{c}$ shows that energy maps in grains 3 and 4 are more or less symmetric with respect to the plane of the GB between them that can be expected due to the symmetry of EGBD systems in the initial systems. However, due to a partial relaxation by a non-symmetrical emission of lattice dislocations (see video files in supplementary material) the exact symmetry is destroyed.

Although no direct comparisons of the energy maps presented in Fig. 3b, c to stress field maps can be done, they can be qualitatively compared. A significant part of the 
GB mesodefect caused by EGBDs is represented by triple junction disclinations, which arise due to a mismatch of GB misorientation changes induced by normal components of the EGBDs [11,14]. A qualitatively similar field of shear stresses is typical for a quadrupole of disclinations (see, for example, [28]).

Thus, the case studies show that by MD relaxation of initial structures constructed by the method proposed, one can obtain model nanocrystals having nonequilibrium GBs and the grains nearly free of dislocations. Also, applying
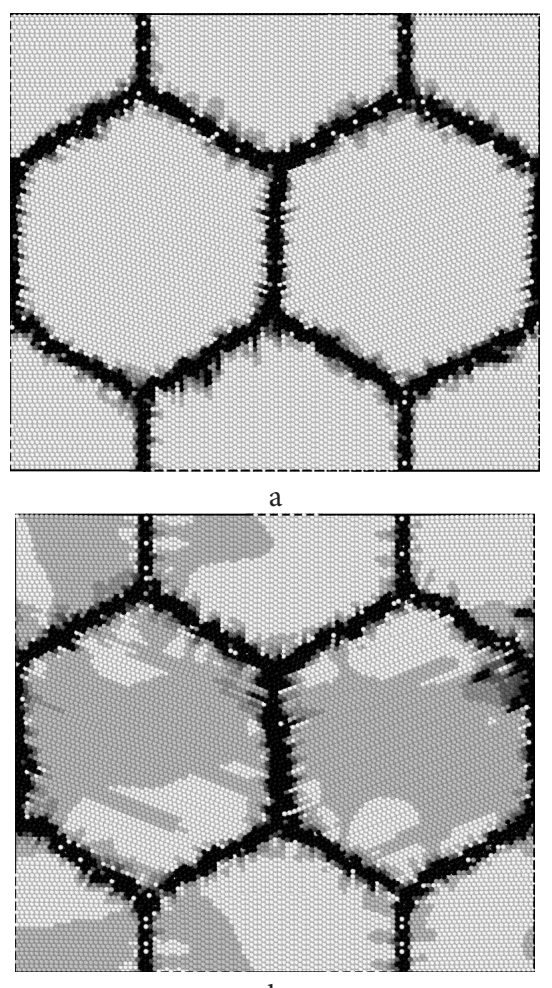

b
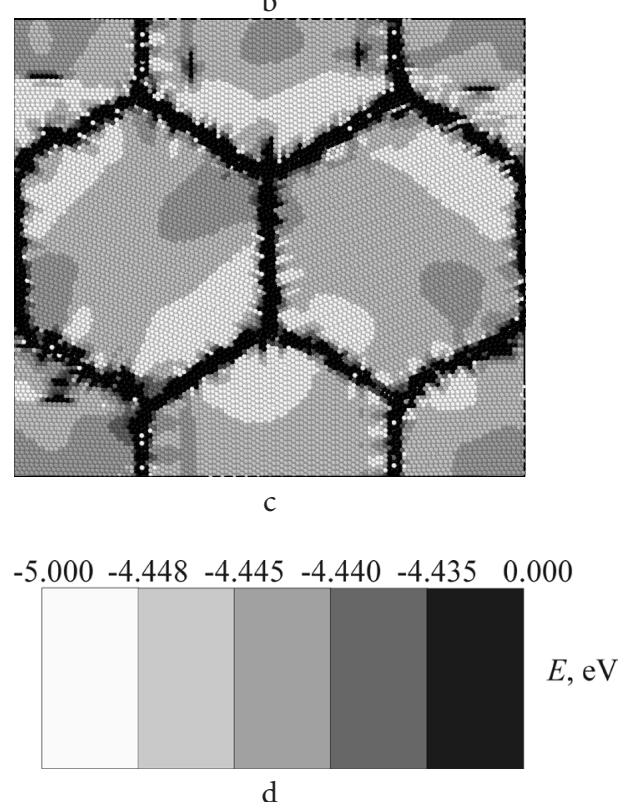

Fig. 3. Atomic energy maps for the relaxed structures of a nanocrystal with equilibrium GBs (a) and with nonequilibrium GBs obtained by MD protocols 1 (b) and 2 (c) and corresponding energy levels (d). different kinds and levels of strains on different grains by means of the method, one can create particular types of mesodefects with different values of strength in GBs to study the role of these mesodefects in the energetics, kinetics and mechanical properties of nanocrystals.

Obviously, the method can be developed for the case of more general, three-dimensional nanocrystals, for example, for those created by Voronoi construction. In this case, however, more complicated transformations including slip on several systems should be applied to each grain.

\section{Conclusions}

We have proposed a method for the construction of computer models of nanocrystalline metals with nonequilibrium GBs, i.e., with GBs containing EGBDs based on shear straining of grains. A case study for [112] columnar nanocrystals has shown that the method allows one to obtain nanocrystals with a desired level of nonequilibrium, which have an enhanced GB energy and long-range internal strains. The method can be generalized for the case of three dimensional nanocrystals.

Acknowledgements. The present work was supported by the Russian Science Foundation (Grant No. 16-19-10126) and by Federal Agency of Scientific Organizations State Assignment (Reg. No. AAAA-A17-117041310213-0).

Supplementary Material. The online version of this paper contains supplementary material (video files demonstrating the processes of $M D$ relaxation) available free of charge at the journal's Web site (www.lettersonmaterials.com).

\section{References}

1. T.P. Darby, R. Schindler, R.W. Balluffi, Philos. Mag. 37, 245 (1978). DOI: 10.1080/01418617808235438

2. R.Z. Valiev, V. Yu. Gertsman, O.A. Kaibyshev, Phys. Stat. Sol. (a) 97, 11 (1986). DOI: 10.1002/pssa.2210970102

3. H. Gleiter. J. Less-Common Metals. 28, 237 (1972). DOI: 10.1016/0022-5088(72)90132-4

4. A. A. Nazarov, A.E. Romanov, R.Z. Valiev, Acta Metall. Mater. 41, 1033 (1993). DOI: 10.1016/09567151(93)90152-I

5. P. H. Pumphrey, H. Gleiter. Philos. Mag. 32, 881 (1975). DOI: $0.1080 / 14786437508221629$

6. I.A. Ovid'ko, A. G. Sheinerman. Philos. Mag. 83, 1551 (2003). DOI: 10.1080/1478643031000088674

7. A. A. Nazarov. Philos. Mag. Lett. 80, 221 (2000). DOI: 10.1080/095008300176191

8. A. A. Nazarov. Philos. Mag. A 69, 327 (1994). DOI: 10.1080/01418619408244347

9. R. R. Mulyukov. Rev. Adv. Mater. Sci. 11, 122 (2006).

10. R.Z. Valiev, A.P. Zhilyaev, T.G. Langdon. Bulk Nanostructured Materials: Fundamentals and Applications. Wiley, Hoboken. (2013).

11. A. A. Nazarov, R. R. Mulyukov. Handbook of Nanoscience, Engineering, and Technology. Eds.W. Goddard, 
D. Brenner, S. Lyshevski, G. Iafrate. CRC Press, Boca Raton. (2002) pp.22-1-22-41.

12. R.Z. Valiev, A.V. Korznikov, R.R. Mulyukov, Mater. Sci.Eng. A. 168, 141 (1993). DOI: 10.1016/09215093(93)90717-S

13. A.P. Zhilyaev, B.-K. Kim, J.A. Szpunar, M.D. Baro, T. G. Langdon. Mater. Sci.Eng. A 391 (2005) 377. DOI: 10.1016/j.msea.2004.09.030

14. V. V. Rybin. Large Plastic Deformations and Fracture of Metals. Metallurgiya, Moscow. (1986) 224 p. (in Russian). [B.В. Рыбин. Большие пластические деформации и разрушение металлов. М.: Металлургия, 1986. 224 с.]

15. A.A. Nazarov, A.E. Romanov, R.Z. Valiev. Nanostr. Mater. 6, 775 (1995). DOI: 10.1016/0965-9773(94)90131-7

16. A. A. Nazarov, A.E. Romanov, R.Z. Valiev., Scripta Mater. 34, 729 (1996). DOI: 10.1016/1359-6462(95)00573-0

17. A.A. Nazarov, Scripta Mater. 37, 1155 (1997). DOI: 10.1016/S1359-6462(97)00230-3

18. A. Hasnaoui, H. Van Swygenhoven, P.M. Derlet. Acta Mater. 50, 3927 (2002). DOI: 10.1016/S13596454(02)00195-7
19. G. J. Tucker, D.L. McDowell. Int. J. Plast. 27, 841 (2011). DOI: 10.1016/j.ijplas.2010.09.011

20. T. J. Rupert, C. A. Schuh. Phil. Mag. Lett. 92, 20 (2012). DOI: 10.1080/09500839.2011.619507

21. T. Shimokawa, T. Hiramoto, T. Kinari, S. Shintaku. Mater. Trans. 50, 2 (2009). DOI: 10.2320/matertrans.MD200810

22. A. A. Nazarov, A.E. Romanov, R.Z. Valiev. Phys. Stat. Sol. (a) 122, 495 (1990). DOI: 10.1002/pssa.2211220208

23. S. M. Foiles, M. I. Daw, M. S. Baskes. Phys. Rev. B 33, 7983 (1986). DOI: 10.1103/PhysRevB.33.7983

24. XMD-Molecular Dynamics for Metals and Ceramics. http://xmd.sourceforge.net/about.html.

25. Home page for RasMol and Open RasMol. http://www.openrasmol.org.

26. A. Stukowski. Modell. Simul. Mater. Sci. Eng. 18, 015012 (2010). DOI: 10.1088/0965-0393/18/1/015012

27. J. D. Honeycutt, H. G. Andersen. J. Chem. Phys. 91, 4950 (1987). DOI: 10.1021/j100303a014

28. D. V. Bachurin, R. T. Murzaev, A. A. Nazarov. Modelling Simul. Mater. Sci. Eng. 25, 085010 (2017). DOI: 10.1088/1361-651X/aa9199 